\title{
EDITORIAL
}

\section{Impaired function in the complex patient with COPD: a matter to be considered}

The very frequent association between chronic obstructive pulmonary disease (COPD) and other chronic disorders sharing common risk factors, ${ }^{1}$ has been widely recognized as a major burden on individuals and healthcare systems. If we exclude lung cancer, the most common co-existing conditions that can be objectively measured in COPD patients are cardiovascular diseases, type II diabetes, skeletal muscle dysfunction and osteoporosis, which are represented in different clusters. ${ }^{2}$

In the last few years the term "comorbidities" has been replaced by "multimorbidities" to better reflect the nature of this link, with clear reference to a shared pattern of metabolic abnormalities, systemic inflammation and defined risk factors that identifies COPD as one component - not necessarily the most relevant - of the clinical phenotype of a chronic complex patient. ${ }^{1}$

In a recent study, Miller et al. demonstrated that cardiovascular diseases and diabetes have the highest impact on several patient-related outcomes in COPD patients, even after adjusting for age, sex and smoking history. ${ }^{3}$ As a matter of fact, the mutual interaction between COPD and cardiac dysfunction is based not only on similar risk factors and pathobiological features such as systemic inflammation, but also on pathophysiological mechanisms such as lung hyperdistension, ${ }^{4}$ which may impair both ventilatory and cardiac function during effort, thus limiting exercise performance both at maximal or submaximal level. In particular, the dynamic hyperinflation on exertion found in COPD patients with different degrees of airflow obstruction, is associated with a reduction of cardiac output during exercise, limiting both venous return and left ventricle filling volume. ${ }^{5}$ On the other hand, it has been shown that airflow limitation could negatively impact exercise capacity and physical performance in patients with chronic heart failure. ${ }^{6}$ Systemic hypertension has been related to the increased inflammatory subset observed in COPD; ${ }^{7}$ the

DOI of original article:

http://dx.doi.org/10.1016/j.rppnen.2015.04.003 correlation between hypertension, higher degree of dyspnea and reduced physical activity has also been reported. ${ }^{8}$ This seems even more important if we consider the effect of hypertension on coronary artery disease progression and left ventricular dysfunction, with worsening in exercise tolerance. ${ }^{9}$ Furthermore, Watz et al. showed that the presence of the metabolic syndrome -where systemic hypertension and diabetes coexist- among COPD patients is definitely associated with impaired level of physical activity. ${ }^{10}$

In this issue of the journal, Da Silva and co-workers conducted a cross-sectional study to investigate to what extent the presence of co-existing hypertension, coronary heart disease and diabetes may affect functional capacity in a cohort of 79 patients with COPD. ${ }^{11}$ These comorbidities have been chosen from others according to their higher prevalence in the population of $\mathrm{COPD}^{12}$ and relevant effect on major outcomes. Patients were classified as having "none", "one", and "two or three" of these comorbidities, and the individual's functional capacity has been measured objectively by means of spirometry and sixminute walked distance (6MWT). As well as the perceived health status, 6MWT decreased progressively from "none" to "two or three" categories (see Fig. 1). Interestingly, the number of comorbidities of interest was independently associated with the submaximal exercise performance after adjusting for main confounders (age, severity of COPD, and score of health status).

These findings need to be discussed in more depth, ideally aspects such as weakness and strength should be addressed and clarified for the readers.

In line with previous literature ${ }^{3}$ authors confirm the lack of association between the degree of airflow obstruction and the number of concomitant disorders. However, this study only provides the evidence about 3 comorbidities, although they are the most important, which relate to the individual's function. Impaired 6MWT and 6MWT-derived variables (speed, work, exercise-induced oxygen saturation) have an additional predictive value of mortality in patients with COPD ${ }^{13}$ however this performance could be influenced by 


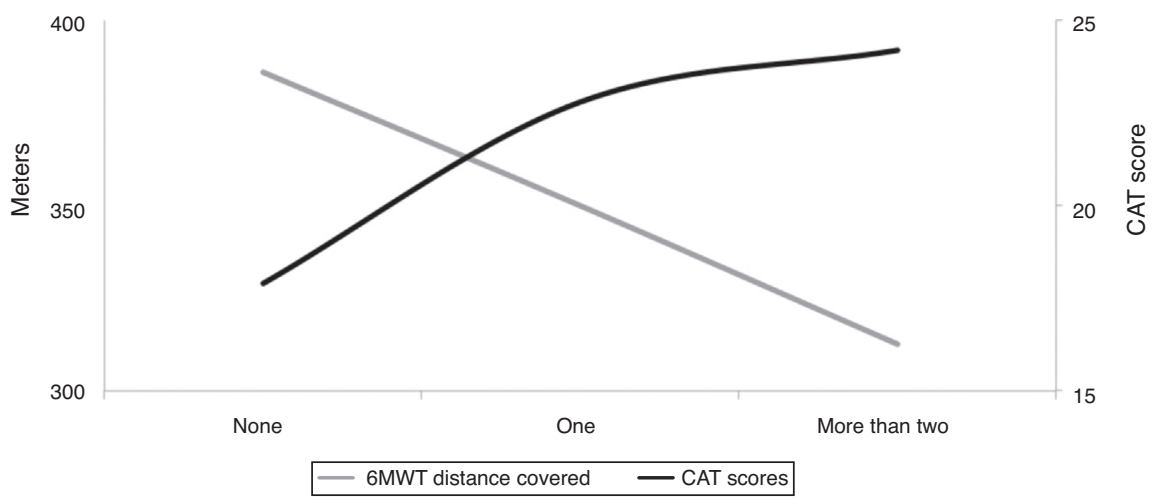

Fig. 1 Relationship between number of the (considered) comorbidities and functional impairment as assessed by health status CAT score (from ref\#11).

other coexisting diseases (i.e. musculoskeletal disorders) and factors that have not been taken into account by the authors. In addition, no information was given about the severity of each of these 3 comorbidities, nor about ongoing therapies for managing them.

We recognize that hypertension, coronary heart disease and diabetes are frequent forms of multimorbidity influencing the individual's function as single diseases or clustering together. ${ }^{3,8,14}$ Indeed, authors have confirmed that these chronic diseases have a negative impact on the exercise capacity, here recorded as both the reduction in meters walked and the increased scores of the items assessing function in the COPD Assessment Test (CAT). Therefore, accurate assessment and count of comorbidities in the characterization of patients with COPD contribute to predicting their outcomes and risk of mortality. ${ }^{12}$

Another problem with the present data might be that the presence of hypertension, coronary heart disease and diabetes was self-reported and/or derived by the assumed medications, there was no objective assessment. Recently, it has been shown that self-reporting may result in underestimation of chronic diseases and multimorbidity. ${ }^{15}$

Walking performance, however, does not exclusively represent the functional capacity of patients with COPD. We can only speculate on whether there is direct correlation between 6MWT and the individual's physical activity (PA), since a good degree of exercise tolerance is pivotal to performing complex kinds of activities in daily living. In COPD subjects, lower levels of PA are associated with higher degree of severity ${ }^{16}$ and a worse prognosis. ${ }^{17}$ A decline to low PA over time is associated with an increased mortality risk in those with COPD but also those without. ${ }^{18}$ This suggests that it is important to assess and encourage PA in the earliest stages of COPD in order to maintain it at the highest possible level, as this is associated with better prognosis. However, PA cannot be derived by the submaximal exercise test in the COPD population, nor did authors measure PA in their study sample. A deeper discussion on the existing links between exercise performance and PA should be taken in consideration in order to understand the extent to which other chronic co-existing diseases might limit function in patients with COPD.

The cumulative effect of multimorbidity in limiting physical performance should be carefully considered when a
COPD patient is assessed and managed, paying particular attention to rehabilitation. To date, pulmonary rehabilitation, including exercise training, has proved to be one of the most effective therapies for COPD patients. ${ }^{19,20}$ Furthermore, recent studies have shown that even changes in the level of activity following rehabilitation have direct proportional effects on major outcomes in these patients. ${ }^{18}$ At present, some retrospective studies have shown worse outcomes (in terms of exercise tolerance, and perceived well-being) in COPD patients with associated heart and metabolic diseases undergoing pulmonary rehabilitation, while others reported the opposite effect. ${ }^{21}$ The reason behind this difference might be the higher degree of impairment in these patients who are generally more dyspnoeic ${ }^{3}$ and less physically active. ${ }^{22}$ However, evidence from available literature is incomplete and there is a need for adjunctive data to understand the effective role of coexisting diseases on rehabilitation outcomes in the population of COPD. For a given different pattern of response to exercise in the complex patients, a tailored intervention could be thus supposed as most important. Although the link between co-existing diseases and reduced performance during pulmonary rehabilitation has been clearly shown, the present study by Da Silva ${ }^{11}$ did not consider this aspect.

In conclusion, taking all the limitations of the present study into account, results corroborate actual debate around the impact of COPD-associated multimorbidity on physical performance and the consequences when a physical therapy is applied to patients. The topic seems to be of critical importance in order to ensure the most effective and personalized treatment for COPD patients referred for a rehabilitation course.

\section{References}

1. Van Remorteel H, et al. Risk Factors and Comorbidities in the Preclinical Stages of Chronic Obstructive Pulmonary Disease. Am J Respir Crit Care Med. 2014;189:30-8.

2. Vanfleteren LE, et al. Clusters of comorbidities based on validated objective measurements and systemic inflammation in patients with chronic obstructive pulmonary disease. Am J Respir Crit Care Med. 2013;187:728-35.

3. Miller J, et al. Comorbidity, systemic inflammation and outcomes in the ECLIPSE cohort. Respir Med. 2013;107:1376-84. 
4. Barr GR, et al. Percent emphysema, airflow obstruction, and impaired left ventricular filling. N Engl J Med. 2010;362:217-27.

5. Tzani P, et al. Dynamic hyperinflation is associated with a poor cardiovascular response to exercise in COPD patients. Respir Res. 2011;12:15.

6. Mentz RJ et al., Clinical characteristics, response to exercise training, and outcomes in patients with heart failure and chronic obstructive pulmonary disease: Findings from Heart Failure and A Controlled Trial Investigating Outcomes of Exercise TraiNing (HF-ACTION), American Heart Journal Volume; 165: 193-99.

7. Divo M, Cote C, de Torres JP, et al. Comorbidities and risk of mortality in patients with chronic obstructive pulmonary disease. Am J Respir Crit Care Med. 2012;186(2):155-61.

8. Hillas G, et al. Managing comorbidities in COPD. Int J Chron Obstruct Pulmon Dis. 2015;10:95-109.

9. Rutten FH, Vonken EJ, Cramer MJ, et al. Cardiovascular magnetic resonance imaging to identify left-sided chronic heart failure in stable patients with chronic obstructive pulmonary disease. Am Heart J. 2008;156(3):506-12.

10. Watz H, et al. The Metabolic Syndrome in Patients With Chronic Bronchitis and COPD. CHEST. 2009;136:1039-46.

11. Da Silva GPF, et al. Exercise capacity impairment in COPD patients with comorbidities. Rev Port Pneumol. 2015.

12. Mannino DM, et al. Prevalence and outcomes of diabetes, hypertension and cardiovascular disease in COPD. Eur Respir J. 2008;32:962-5.

13. Andrianopoulos V, et al. Prognostic value of variables derived from the six-minute walk test in patients with COPD: Results from the ECLIPSE study. Respir Med. 2015.

14. Barnett K, Mercer SW, Norbury M, Watt G, Wyke S, Guthrie B. Epidemiology of multimorbidity and implications for health care, research, and medical education: a cross-sectional study. Lancet. 2012;380(9836):37-43.
15. Siebeling L, Puhan MA, Muggensturm P, Zoller M, Ter Riet G. Characteristics of Dutch and Swiss primary care COPD patients baseline data of the ICE COLD ERIC study. Clinical Epidemiology. 2011;3:273-83

16. Watz $\mathrm{H}$, Waschki B, Meyer T, Magnussen $\mathrm{H}$. Physical activity in patients with COPD. Eur Respir J. 2009;33(2):262-72.

17. Garcia-Aymerich J, et al. Regular physical activity reduces hospital admission and mortality in chronic obstructive pulmonary disease: a population based cohort study. Thorax. 2006;61:772-8.

18. Vaes AW, et al. Changes in physical activity and all-cause mortality in COPD. Eur Respir J. 2014;44:1199-209.

19. Lacasse $Y$, et al. Meta-analysis of respiratory rehabilitation in chronic obstructive pulmonary disease. A Cochrane systematic review. Eura Medicophys. 2007;43:475-85.

20. Gloeckl R, et al. Practical recommendations for exercise training in patients with COPD. Eur Respir Rev. 2013;22(128):178-86.

21. Franssen FEM, Rochester CL. Comorbidities in patients with COPD and pulmonary rehabilitation: do they matter? Eur Respir Rev. 2014;23:131-41.

22. Crisafulli $E$, et al. Role of comorbidities in a cohort of patients with COPD undergoing pulmonary rehabilitation. Thorax. 2008;63:487-92.

R. Tonelli ${ }^{a}$, E.M. Clini ${ }^{a, b, *}$

${ }^{a}$ DU of Medical and Surgical Sciences, University of Modena-Reggio Emilia, Italy

b Ospedale Villa Pineta, Pavullo n7F (Modena), Italy

* Corresponding author. University of Modena-Reggio

Emilia, Ospedale Villa Pineta, Pavullo n/F (Modena), Italy. Tel.: +390536 42039; fax: +39053642039.

E-mail address: enrico.clini@unimore.it (E.M. Clini). 